\title{
On the limitations of analysing worst-case dynamic energy of processing
}

\author{
Jeremy Morse, Steve Kerrison and Kerstin Eder \\ University of Bristol
}

September 17, 2018

\begin{abstract}
This paper examines dynamic energy consumption caused by data during software execution on deeply embedded microprocessors, which can be significant on some devices. In worst-case energy consumption analysis, energy models are used to find the most costly execution path. Taking each instruction's worst case energy produces a safe but overly pessimistic upper bound. Algorithms for safe and tight bounds would be desirable. We show that finding exact worst-case energy is NP-hard, and that tight bounds cannot be approximated with guaranteed safety. We conclude that any energy model targeting tightness must either sacrifice safety or accept overapproximation proportional to data-dependent energy.
\end{abstract}

\section{Introduction}

A significant design constraint in the development of embedded systems is that of resource consumption. Software executed on embedded hardware typically has very limited memory and computing performance available, and yet must meet the requirements of the system. To aid the design process, analysis tools such as profilers or maximum-stack-depth estimators provide the developer with information allowing them to refine their designs and satisfy constraints.

A less well studied constraint is the limited energy and power budgets that apply to deeply embedded systems. This is a contemporary challenge for the proliferation of such devices, particularly those that operate in isolated environments, with limited energy availability and where the processor is the largest consumer of energy in the system. A typical example would be a wireless sensing device powered by battery, that has long intervals between wireless communication, such that processor activity dominates the system's energy consumption. The device may need to operate for a minimum period without the battery being replaced, therefore it has a total energy budget.

Other examples are systems dependent on energy harvesting, or systems with low thermal design points and thus have a maximum power dissipation level that may be independent of the total energy they consume. Whether these constraints are satisfiable can be examined with software analysis tools, and several techniques have been developed that allow the estimation of software's energy consumption [23, 11, 22, 15, 41].

Within energy estimation, Worst Case Energy Consumption (WCEC) has been explored, determining the maximum amount of energy that can be consumed during the execution of the 
software. In this paper, we shall study the calculation of worst-case energy, considering only the effects that different software and inputs can have on a system. The objective is to determine whether it is possible to establish an upper bound on energy that is tighter than over-estimating by, for example, using a maximum activity factor. Such a factor may be unachievable during the execution of a real program, because an operand value that triggers the highest energy consumption in one instruction may, through data dependency and other constraints, preclude subsequent instructions from consuming their maximal energy [27].

Energy is the integral of power over a given time interval. The power dissipation of a processor can be apportioned in two parts: static and dynamic. Static power or leakage is the power dissipated for as long as the component is turned on, irrespective of its internal state or any changing inputs and outputs. Dynamic power or switching activity refers to power dissipation due to changes within the processor: the clock tree, switching of gates and charging of data buses, which all consume energy. We express these more formally in Section 3. Analysis of worst-case instantaneous dynamic power has been well studied in the literature, but here we consider worst-case energy, i.e. the integral of power over a program execution.

Estimating worst-case energy for a particular program requires the computation of these two distinct contributions to power dissipation. Static power is constant in a stable operating environment (for example voltage, frequency and temperature), therefore energy consumption due to static power is proportional to program execution time. Numerous techniques have been developed by the Worst Case Execution Time (WCET) community to address this matter [42]. Dynamic power, however, has received much less attention. Several models of how systems consume energy have characterised the dynamic power only for specific inputs, averaged over all inputs, assumed the upper bound of dynamic power for each instruction [15, 41] or assumed no dynamic power at all [11].

In this paper we demonstrate that for the proportion of dynamic energy that is due to switching caused by operand values, the calculation of the worst-case input to a software execution is an NP-hard problem, and further, that this quantity cannot be approximated to a useful factor. Our proof applies to processors in general, but in practice this proportion of data dependent energy may be small. We show that on an example processor, the Xcore XS1-L [26], a cacheless deeply embedded microprocessor with time-deterministic instruction execution, the proportion of energy that is infeasible to analyse contributes approximately half of the processor's dynamic power.

The rest of the paper is structured as follows: in Section 2 we examine the current state of energy estimation, and related work. In Section 3 we demonstrate the variation in dynamic power due to switching caused by operand values, using the Xcore processor as a case study, and consider this in the context of other embedded processors as well as more complex devices. Section 4 formalises the problem that we are dealing with, which is shown to be NP-hard in Section 5, and in Section 6 we demonstrate that the problem cannot be effectively approximated. We discuss the results in Section 7, including system scopes for which accurate prediction of dynamic power variation due to operand values should be considered, versus those where it is less of a concern. Finally, we draw conclusions in Section 8, with an outlook on future work.

\section{Related work and background}

This section identifies existing techniques for determining the energy consumption of software, techniques for determining the maximum amount of energy a program can consume, and the theoretical definition of the MAXSAT problem for use in later proofs. We then go on to analyse the WCEC problem in greater detail. 


\subsection{Energy estimation techniques}

Given the high complexity of microprocessors, energy analysis based on hardware designs tends to be resource intensive, and requires access to proprietary data and tools. Research has instead focused on using empirical techniques to model how processors consume energy. These models can then be used to estimate the consumption of a real-world system.

One of the most popular techniques is the instruction level energy model [39]. Various test patterns of instructions are executed on a processor and their power empirically measured, leading to a model of per instruction energy costs and the dynamic cost of switching between different instructions. Simulating an instruction sequence, or interpreting a trace of an execution, can then be combined with this energy model to produce a cost value for the execution. [36] extend this model to include the costs of circuit switching in instruction operands. These costs include the amount of switching occurring on data buses supplying input operands to an instruction, and the switching on the output when a result is written back to the register file.

Further modelling techniques for dynamic power go beyond the core part of the processor, such as analysing flash memory [29], caches [7] and DRAMs [20]. High performance processors feature hardware-provided counters that record metrics such as cache hit rates, which can be used by appropriately parametrised energy models [32]. The energy consumption on the buses to these components can also be influenced by data values and can be modelled accordingly.

In this paper, however, where deeply embedded devices without such features are the focus, we choose to only examine the dynamic power attributable to the core part of the processor. In larger, more complex processors, the dynamic power due to operand values that we explore remains valid, but is a smaller proportion of the total power dissipation, and therefore may be a lower priority for analysis or optimisation.

\subsection{Worst Case Energy Consumption (WCEC) analysis}

WCEC is a form of energy estimate, where the aim is to find the maximum amount of energy that a piece of software will consume, without needing to execute that software. The problem is thus made of two parts: modelling the energy consumption of the software under test, and searching for the execution of it that will lead to the greatest amount of energy consumed. This problem is defined in a similar way to the Worst Case Execution Time (WCET) problem [42] where the execution time of software is modelled, and then the longest possible path found. However, the techniques required to obtain a solution have a number of differences.

The first publication to provide a technique for computing the WCEC of software was by [15], where upper bounds on the energy consumption of several programs were inferred using energy models of software basic blocks and an ILP solver to find a maximal path through the program. The authors additionally debunk the suggestion that the execution path consuming the most time is always the path that also consumes the most energy. With regards to dynamic power, the authors assume maximal circuit switching on every clock cycle but model power management techniques within the processor such as clock gating to create a realistic energy model. The dynamic power of switching due to operand values is not specifically considered, and indeed the authors show that that its contribution of dynamic power to overall energy is low, thus their approximation does not introduce significant imprecision. We address the contribution of operand values to dynamic power in Section 3.

Resource analysis techniques that extract cost relations from programs have been employed to analyse energy consumption bounds [23, 11]. The costs used in these analyses represent energy consumption and are based on models that provide a single energy cost per instruction, obtained by averaging the energy measured from processing random data, constrained to yield valid operands for the respective instruction [18]. However, bounds obtained in this way cannot 
be considered safe, as executions would exist where the energy from operand values exceeds the average case.

More recently, [41] have presented techniques for estimating over and under approximations of WCEC through implicit path enumeration and genetic algorithms, respectively. They do not, however, comment on dynamic power at all: their absolute instruction energy model appears to assume maximum switching for each instruction cost. Their relative energy model does not consider real energy costs, instead estimating the difference in energy consumption between instructions, again with no explicit consideration of dynamic power.

Both Jayaseelan and Wägemann identify inefficiency as being a reason why they cannot compute accurate switching activities for circuits. As we will show in this paper, the problem is infeasibly complex under the $\mathrm{P} \neq \mathrm{NP}$ assumption.

\subsection{Existing complexity results}

Switching activity is a matter studied in detail by the VLSI community for circuit design, as the maximum instantaneous switching in a circuit can affect the power supply requirements [28]. This problem has been shown to be NP-hard [8] and numerous techniques have been developed to estimate of the worst-case power consumption [12], allowing maximum power analysis.

Power estimation itself does not directly correspond with energy estimation. The objective of WCEC is finding the maximal amount of circuit activity over a time interval, rather than the instantaneous maximum, which itself may be incompatible with the circumstances that lead to maximum energy. In particular, software requires that computations be consistent with past inputs, creating additional constraints and dependencies.

Switching between instructions is a notable contributor to energy consumption, which can be controlled through the order in which instructions are executed. Techniques have been developed to reduce consumption through instruction scheduling [31], but this is known to be an NP-hard problem. Instruction scheduling uses pre-computed costs of switching between instructions to determine an optimal static schedule. It does not consider the operands to instructions or any cost that does not have a fixed value.

None of these complexity results are directly applicable to the estimation of energy in datadependent switching during software execution. To the best of our knowledge, we believe this is the first work to consider data-dependent switching costs.

\subsection{Maximum satisfiability}

Part of our proof in this paper relies on demonstrating that an NP-hard problem can be represented within the problem of calculating the amount of circuit switching in a program. We therefore assume the reader is familiar with the SAT problem (a full treatment of which can be found in [33]), and remember the definition of the MAXSAT form of the problem, which we embed within operand value switching in Section 5.

Briefly, the Maximum satisfiability problem "MAXSAT" [3, pp.613-631] takes a set of Boolean variables, constraints on their values in the form of a set of clauses, and finds the variable assignment that makes the maximum number of clauses true. Unlike SAT, not all clauses need to be true. MAXSAT is known to be NP-hard.

Formally, following the presentation of [16], define $L$ to be a set of literals, and $C$ a set of disjunctive form clauses:

$$
\begin{aligned}
L & =\bigcup_{i>0}\left\{x_{i}, \bar{x}_{i}\right\} \\
c \in C, c & =\left\{l_{1} \vee \ldots \vee l_{n} \mid l_{i} \in L\right\},
\end{aligned}
$$


where each $x_{i}$ is a Boolean variable. A truth assignment defines each $x_{i}$ or its negation to be true. A clause is deemed to be satisfied if at least one literal in the clause is assigned true. A MAXSAT problem is a set of literals and set of clauses $\langle L, C\rangle$, such that the solution is the truth assignment that causes the maximal number of clauses to be satisfied.

\subsection{WCEC background}

The worst-case energy consumption problem goes beyond the worst-case execution time problem, because the execution time of a single instruction is largely independent of its input data. This is because timing variability has mostly been eliminated "by design" through the use of synchronous logic and the limited propagation time associated with executing individual instructions.

Despite this, there are scenarios where WCET is subject to timing anomalies [25]. These arise when a seemingly shorter execution path later results in an overall increase in time. A cache miss may cause the worst execution time locally, but this state may later preclude a subsequent scenario that, with a global view, would in fact be the worst-case.

A comparable anomaly in energy is two equal-time paths, where one path contains an instruction known to produce the worst-case energy. However, the input data that reaches that instruction does not achieve that worst-case, due to transformations performed upon it by previous instructions in the path. Instead, the sum of instruction energies on the alternate path is higher than the sum of energies on the apparent worst-case path.

In real-time embedded systems, timing-predictable processors execute instructions within a fixed number of clock cycles, irrespective of the data the operation works on. This is particularly beneficial to WCET analysis, which can then focus on identifying the worst-case execution path which is determined by the control flow, rather than by the data flow of the computation. More advanced micro-architectural features, such as early-out of operations, or cache hierarchies, provide higher average performance at the cost of predictability. This makes WCET analysis far more challenging, as tight bounds firmly rely on timing predictability of the target architecture [37]. However, even operations that have a variable execution time, such as serialized integer multiply and divide, or floating point operations, can be quantized by the processor's clock period into a tractable number of discrete possibilities. The range may be in the order of tens, hundreds, or thousands of cycles, depending on the type of operation. This extends into other architectural features, such as caches and branch predictors, which although more complex to analyse, can still be quantized.

Energy depends on both the execution time and the power dissipation of the operation. Power is not quantized in terms of the clock period, but could be considered in terms of the number of transistor and interconnect state changes (i.e. switches) that may take place during an operation, depending on the data to be processed. The number of possible power dissipation levels is thus the size of the powerset of the number of transistors in the device. This is several orders of magnitude larger than the number of timing possibilities that need to be explored by WCET analysis. This view is itself simplified, as it does not consider the continuous variations in temperature and voltage that a device will be affected by.

For the techniques that are used in WCET to be directly transferable to WCEC, a set amount of energy per operation would need to be specified and realised in hardware, similar to specifying and ensuring, through timing analysis at design time, that each operation fits into a fixed number of clock cycles. Consider the converse: A processor that presents a similar WCET analysis difficulty would be an asynchronous design, where the precise execution time is a non-trivial function of an operation's input data. Such devices may have an average delay, but actual performance or tight bounds for a given use case may be harder to determine [17].

Energy estimates can also have varying degrees of accuracy. The strongest is to determine the exact WCEC, which consists of a program trace causing the maximum amount of energy that 
can expended, and the input that causes the trace. The computational difficulty of finding the exact worst-case has fuelled interest in approximation algorithms [40] that guarantee to find an example of the energy consumed by the program that is within some factor of the worst-case. This estimate can be considered to be an approximation with a limited amount of uncertainty, or alternately the approximate energy plus the uncertainty factor can be used as a safe upper bound on the true WCEC. If the uncertainty factor is low, this may be sufficient to prove that a design constraint is met.

Finally, upper bounds can be derived from the structure of the program itself through very coarse over-approximation, for example by assuming a program always exhibits the maximum activity factor. Such over-approximation is the most inaccurate of such techniques, but also the most feasibly achievable. The distinction between approximation algorithms and coarse overapproximation is that the former has a definite relationship with the exact WCEC, while the latter is based for example only on the length of the program, and provides no guarantees on what the exact WCEC may be.

This paper addresses the first two forms of WCEC estimate and demonstrates that they are not feasibly computable, leaving only the coarse over-approximation as a viable technique in practice.

\section{Circuit switching on Xcore}

Prior WCEC papers have relied on the suggestion that the variation in dynamic power is small in relation to other energy costs in a processor, at approximately $3 \%$ [38], therefore a conservative average-based model may be suitable. Other work has presented a mixed picture: [35] found that the switched capacitance (i.e. switching cost) of a StrongARM processor had little variance across applications, suggesting that switching costs contribute little to overall program energy; while [2] observe that data switching accounts for up to $50 \%$ of processor core energy.

Here, we affirm two properties of dynamic power dissipation in a processor by analysing the Xcore [26] XS1-L. First, that dynamic power due to operand values can be high, and second, that this cost can vary significantly.

\subsection{Defining power dissipation in a micro-processor}

The energy, $E$, of an electronic device is the integral of its power dissipation, $P$, over a given time period, $T$ :

$$
E=\int_{t=0}^{T} P(t) d t .
$$

Power is an instantaneous measure of the rate of work. Typically, this is sampled repeatedly in order to discretise the integral, or the power is averaged, simplifying the equation to $E=P \times T$. In digital devices such as processors, the total power dissipation of the device, $P_{t o t}$ is typically apportioned into two additive parts, termed static and dynamic, denoted here as $P_{s}$ and $P_{d}$ respectively:

$$
P_{t o t}=P_{s}+P_{d}
$$

Elaborating on these, static power is determined by the operating voltage, $V_{d d}$ of the device and $I_{\text {leak }}$, the leakage current present, which is itself dependent upon physical characteristics such as operating temperature, transistor feature size and the manufacturing process that is used [4], yielding an exponential equation [10]:

$$
I_{l e a k}=W I_{s} e^{\frac{V_{t h}}{V_{d d}}}
$$


for transistor width, $W$, sub-threshold current, $I_{s}$, as well as the voltage threshold and operating voltage, $V_{t h}$ and $V_{d d}$, respectively. However, a linear approximation is sufficient for the normal operating range of many devices, therefore a simplified representation of static power is:

$$
P_{s}=V_{d d} I_{l e a k}, \quad \therefore \quad P_{s} \propto V_{d d}
$$

Dynamic power is dependent upon the capacitance of the components that are being switched, $C_{s w}$, as well as the operating voltage and the frequency of switching, $f$. In a processor, $f$ is governed by the clock frequency. The proportion of the device that is switching is dependent upon the instruction and data being executed and related changes in state. This is represented by an activity factor, $\alpha$, where each instruction or action performed by the processor may have a different $\alpha$.

$$
P_{d}=\alpha C_{s w} V_{d d}^{2} f, \quad \therefore \quad P_{d} \propto V_{d d}^{2}
$$

There is a quadratic relationship between voltage and dynamic power. The necessary operating voltage is approximately linearly proportional to the operating frequency in processors operating above the threshold voltage of their transistors [19].

\subsection{Apportioning dynamic power}

The power dissipation of a single instruction is typically expressed as the average power observed during the execution of that instruction. When considering a model of power per instruction, it is important to calculate an appropriate $\alpha$ per instruction, or some equivalent by abstraction. However, the instruction is not the sole influence upon the $\alpha$ value. The operands supplied to the processor's functional units (for example, arithmetic unit), will affect the amount of switching. This includes changes to the input and output of the functional unit, as well as internal switching within the unit as the new result is computed. As such, one instruction may have a range of possible $\alpha$ values that are dependent on the input data.

Prior work [15] has suggested that this variation in $\alpha$ is small and therefore not significant enough to consider when constructing a worst-case energy model. However, we demonstrate that variation in input data can be responsible for as much as $42 \%$ of a core's power dissipation and thus becomes a relevant contributor to the model. This is pertinent to systems with minimal additional components, such as those that are deeply embedded, where the processor is the major consumer of energy. In larger, more complex systems, with multi-layer memory hierarchies, many external peripheral devices and several power supplies, the variation in total system energy due to operand values is proportionally smaller.

Internal processor data buses are one of the largest contributors to dynamic power in an embedded processor. These buses interconnect various internal units, and so changing values on these buses indicate the charge or discharge of connections between a number of gate inputs and outputs, which may have different loads depending on their fan-in or fan-out and connection length. The [36] energy model explores this and discovers that approximately $20 \%$ of overall processor power can be attributed to the Hamming distance between transitions on buses.

To determine the dynamic power cost on our target device (the Xcore XS1-L), we performed experiments in the manner of [36]. For a set of instructions, we tested every combination of input operand values from zero to 255 for each operand, creating a sequence of tests, $\mathbb{P}$. We alternate between instructions with this data set and all-zero operands, to ensure we measure the Hamming weight on each cycle. On the Xcore, this instruction sequence is achieved with four parallel, tightly coupled threads that are issued into the pipeline round-robin by the processor's deterministic hardware scheduling. Alternating threads perform the desired operation on all-zero data and the operand values under test. Unrolled sequences are used to minimize noise from 


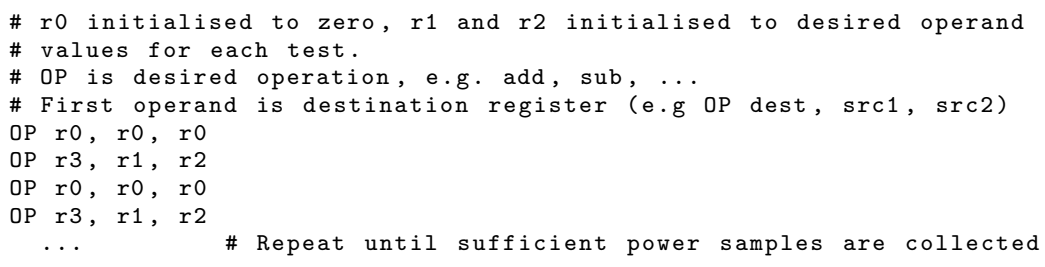

Listing 1: Example trace of instructions.

loop instructions that we do not want to measure. In a single-threaded in-order processor, a similar sequence can be achieved with one thread.

An example instruction trace is given in Listing 1. This method effects a change in energy consumption due to operand values along the core's internal datapath, starting at the reading of the register file, progressing through the functional units of the core, then once again to the register file for write-back. The effect of memory access does not fall within the scope of this work, although we acknowledge the impact of this in Section 2.5 and discuss the proportional contributions at the system level in Section 7.

The Xcore is a cache-less multi-threaded processor with time-deterministic execution, therefore there is no complex memory hierarchy to influence power or time, and all of the instructions that we test take the same, constant execution time. Test sequences were constructed in such a way to ensure we exercised the datapath between the register file and functional units in every instruction cycle. Tests are applied in a single-core context, with no inter-thread communication. Although the processor has 32-bit operands, exhaustive testing over 8-bit data presents datasensitive energy patterns that are sufficient to expose the behaviours of interest to this work. For wider data, these patterns repeat, potentially with larger patterns becoming apparent. This is also evident for narrower data, where patterns are still observable in smaller subdivisions of the figures that we present in the following subsection. As such, using the full width of the processor is not necessary to analyse the problem that we present in this paper.

The device is operated with a $1.0 \mathrm{~V}$ core power supply and $500 \mathrm{MHz}$ clock frequency. Power is sampled at the $3.3 \mathrm{~V}$ input to the DC-DC converter that supplies the cores and is done using a vendor-supplied sampling and debug device that uses a shunt resistor to determine current. The tests are each run repeatedly for a $0.5 \mathrm{~s}$ duration in order to acquire several thousand power samples, then taking the average.

The device under test is the XS1-A16A-128, a dual-core component, tested with single-core code. The device has two discrete cores in a single processor package, with voltage regulators and unused analogue peripherals being the only shared components also measured. We assume the peripheral components consume negligible energy when unused, and that the power supply contribution will be proportionally similar on single core, assuming the DC-DC is re-selected to achieve the same efficiency at a lower current.

We remove the additional energy consumption that would not be present if a single-core version of the component were to be used. We refine the total power, $P_{t o t}$, by considering the distribution of power between the two cores. This is established through a simple step, measuring the total power of the dual-core device when idle, represented as $P_{t d u a l}$, then enabling us to ascertain $P_{\text {tsingle }}$, representing a single core:

$$
P_{\text {tsingle }}=\frac{P_{\text {tdual }}}{2}
$$

Following this, the dynamic power dissipation due to operand values is isolated. Executing instruction power tests on one core, leaving the remaining core idle, produces a sequence of test 
results, $\mathbb{P}=\left\{P_{0}, \ldots, P_{n}\right\}$. We define the dynamic power contribution of the lowest and highest power test cases as $P_{d \min }$ and $P_{d \max }$ respectively, and the dynamic power range, $P_{d r n g}$ :

$$
\begin{aligned}
P_{d m i n} & =\min (\mathbb{P})-P_{\text {tdual }}, \\
P_{\text {dmax }} & =\max (\mathbb{P})-P_{\text {tdual }}, \\
P_{\text {drng }} & =P_{\text {dmax }}-P_{\text {dmin }},
\end{aligned}
$$

or as a percentage contribution to total processor power:

$$
\frac{P_{x}}{P_{\text {tsingle }}+P_{x}}, \forall x \in\{d \min , d \max \} .
$$

We observe for the device under test that $P_{\text {tdual }}=328 \mathrm{~mW}$ and therefore $P_{t s i n g l e}=164 \mathrm{~mW}$. Any additional power observed during tests is used to determine how much dynamic power variation is possible for the set of input values tested. This is not solely static power, because even at idle, switching in components such as the clock tree is taking place, contributing to dynamic power. Thus, the difference in power observed during instruction and data tests is not the total dynamic power contribution, but does establish the degree of variation in dynamic power that can take place, and what proportion of total core power this amounts to.

\subsubsection{Heat-map observations}

To aid analysis of the results of these experiments, we present a series of "heat-map" figures, showing measured dynamic power in colour, and with operand (input) and result (output) Hamming weights in greyscale. These plots use measurements from tests of the add instruction, although similar data-dependent behaviours were observed in other instructions.

Figure 1 shows total dynamic power for add with all combinations of two 8-bit operands. The diagonal striping indicates a strong correlation with the number of bits set to 1 in the result of the computation. This is observable due to alternating between test add operations and operations with all-zero inputs and outputs. The Hamming weight of the result values are shown in Figure 2. This is determined to represent $4.4 \mathrm{~mW}$ per output bit set in respect to the data from Figure 1. Using the Hamming weight range as a scale from zero $\mathrm{mW}$ to the maximum $\mathrm{mW}$ observed, the Hamming weight predicts dynamic power with a mean error of $3 \mathrm{~mW}$.

Subtracting the calculated switching power per result bit from the original dynamic power measurements gives Figure 3. This reveals a second pattern of vertical and horizontal striping that was previously obscured by the dominant effects of the Hamming weight of the output value of the add operation. Intuitively, this corresponds to the Hamming weight of both input operands, demonstrated in Figure 4. We determine this to be $1.3 \mathrm{~mW}$ per input bit set. The input Hamming weight predicts power in Figure 3 with a mean error of less than $1 \mathrm{~mW}$. Repeating this process and subtracting the calculated power per input bit gives Figure 5, which closely corresponds to the pattern observed for the Hamming weight shown in Figure 2, as previously stated.

Finally, by subtracting the dynamic power of both the input and output bits produces Figure 6 , which shows that the remaining variation in dynamic power is an order of magnitude lower than the effect of these Hamming weights, ranging from $12 \mathrm{~mW}$ to $0 \mathrm{~mW}$. Expressed as a series of matrix operations, where $P$ is the measured dynamic power and the input and output Hamming weights are presented as $H_{i}$ and $H_{o}$ respectively, the remaining unaccounted for dynamic power, $D$ of Figure 6, is:

$$
D=P-\left(H_{i} \cdot 1.3\right)-\left(H_{o} \cdot 4.4\right) \quad \mathrm{mW} .
$$

In a real-world program, instructions would not be interleaved with the loading of zero-value operands, as was done in our experiments. However, with each new instruction, a Hamming 


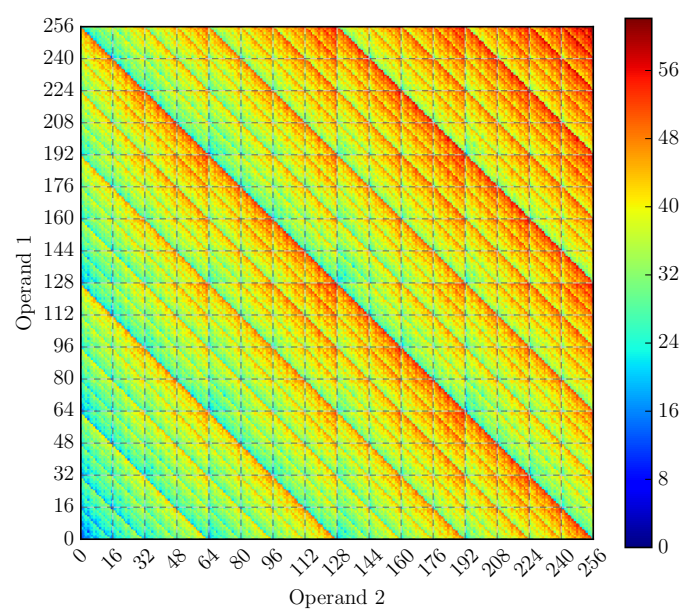

Figure 1: Dynamic power in milliwatts for add, over a range of input operand values.

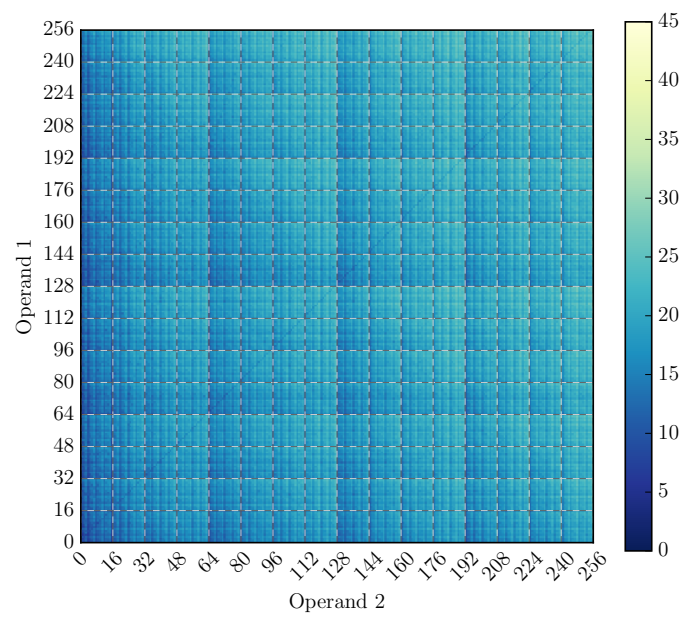

Figure 3: Dynamic power in milliwatts for add, with the output value cost subtracted, at $4.4 \mathrm{~mW}$ per bit.

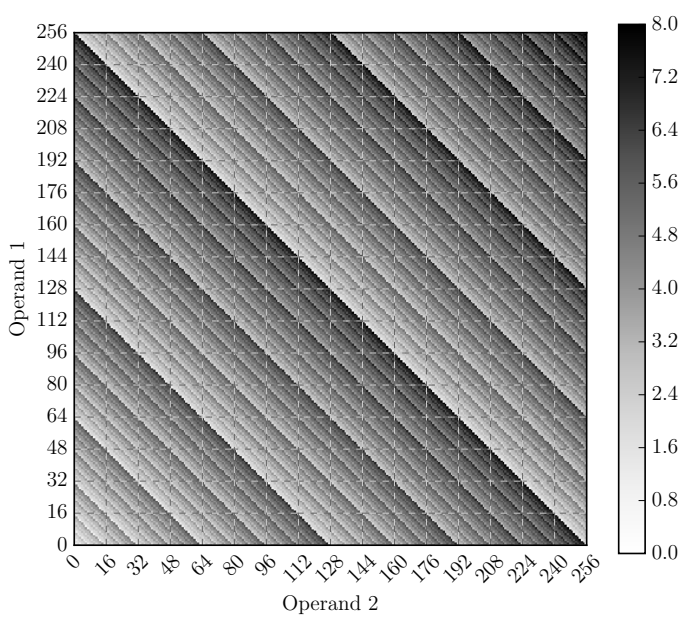

Figure 2: Hamming weight of the output value from performing add, in number of bits set.

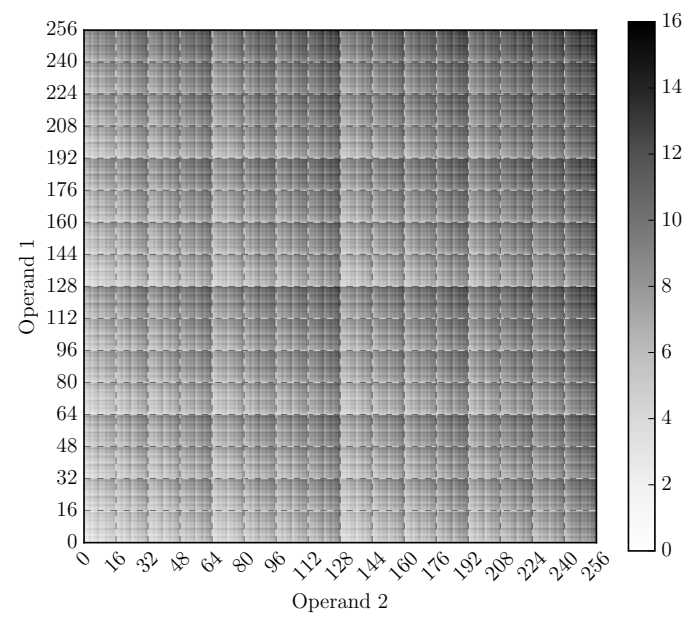

Figure 4: Hamming weight of both input operands to add, in number of bits set. 


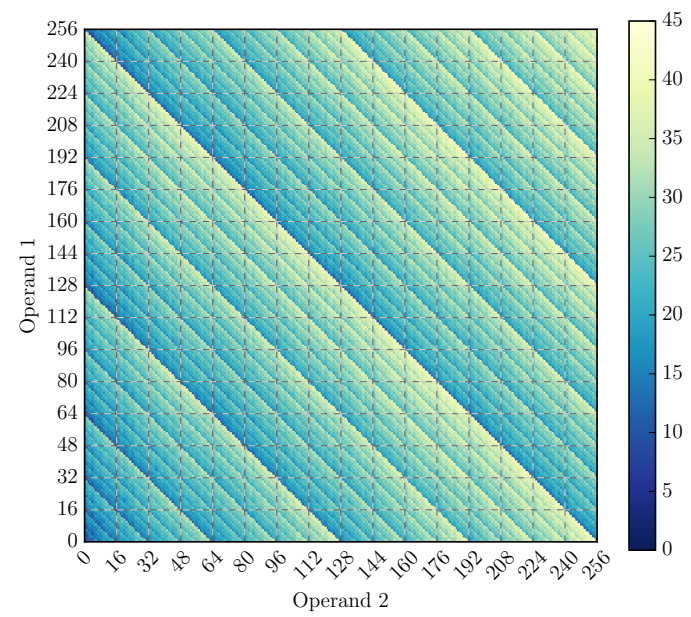

Figure 5: Dynamic power in milliwatts for add instruction, with input datapath cost subtracted (assuming $1.3 \mathrm{~mW}$ per bit).

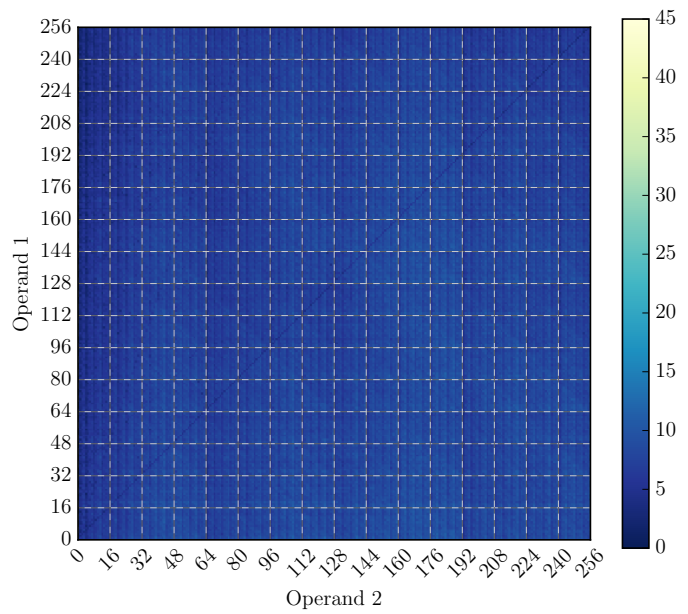

Figure 6: Dynamic power in milliwatts for add instruction, with operand and result value costs subtracted.

distance would be present between the previous and current input values, as well as the previous and current output value. As such, the properties described here naturally translate from Hamming weights into Hamming distances.

\subsubsection{Dynamic power range due to operand values}

For the add instruction, $P_{d \min }=34 \mathrm{~mW}$ and $P_{d m a x}=96 \mathrm{~mW}$, giving $P_{d r n g}=62 \mathrm{~mW}$. Using Eq. (10), this demonstrates that for add, a range of $17-37 \%$ of the core's power dissipation is governed by operand values. This is a twenty percentage point range due to operand values. In a system where processor power is significant, this is a substantial variation, inaccurate predictions of which may be undesirable or unsafe.

Across all of our experiments, the $P_{d \max }$ observed was $123 \mathrm{~mW}$, caused by the sub instruction. This is due to sub producing a negative two's complement output that results in all bits being set in the output operand, causing maximal Hamming distance in the output datapath. However, compared to $P_{\text {tsingle }}$ this means that on the Xcore, dynamic switching contributes as much as $42 \%$ of the total processor power, and that over two-thirds of this contribution is due to operand values. Similar work for 8-bit AVR [30] shows dynamic power making up $15 \%$ of processor power, which is lower than the Xcore, most likely due to its narrower data width but also due to a range of other architectural and manufacturing differences. The AVR, like the Xcore, is cache-less and a candidate for use in deeply embedded systems. Further processor examples include variants of ARM cores in the Cortex-M series, which do not typically include complex memory subsystems [1].

This data demonstrates that, at least on the Xcore and the AVR, the contribution of dynamic power to the full processor cost is non-trivial, and certainly a significant contributor to calculating the worst-case energy in deeply embedded programs. We also observe that the result values for our particular processor are the most significant contributor to dynamic power. This is still input data dependent, because the result value is a transformation of an instruction's input. For simplicity we focus only on the most significant component, the result value, in subsequent sections: specifically, the Hamming distance between the output values of operations across 
subsequent clock cycles. We briefly discuss other components in Section 7 .

\subsection{Summary and Discussion}

With regard to prior work that analyses the significance of dynamic power in software execution, we have demonstrated that on the Xcore dynamic power can be a large proportion of overall energy consumption by the processor, but cannot discount prior work that found little contribution on other platforms. This suggests that dynamic power contribution can be significant, but that it varies from processor to processor. It should also be considered that in real programs, sequences of instructions are unlikely to yield operand values that produce the worst-case for each instruction. In other work [30] we have found energy consumption can vary up to $7 \%$ in two benchmarks on Xcore depending on their inputs, and up to $9 \%$ for the same benchmarks on AVR. However, our intent is to examine the feasibility of finding a bound that is both safe and tight, avoiding methods with less than absolute certainty.

The system context should also be considered, for two main reasons. Firstly, a large system, for example that features a display and backlight component, will have its total energy consumption dominated by these over all other components [5]. Looking beyond embedded systems, large multi-core processors such as the Xeon Phi [34] consume significantly more energy in caches and memories than in computation. This will of course significantly reduce the impact of any variation in processor core's energy. Secondly, the type of system and its performance requirements will influence processor choice, and the amount of power variation of the chosen processor will determine whether it is necessary to consider it. If this is the case, the computational workload placed upon the system will then determine how much each part of the processor is exercised. It is shown in [13] that both processor choice and workload change how processor subcomponents such as the register file and functional units contribute to total energy consumption.

With this in mind, we observe that consideration of dynamic power caused by operand values is most relevant for real-time, deeply embedded applications. Such applications typically have energy budgets as a primary concern, have some non-trivial processing task that requires a microcontroller, but do not use a large processor featuring caches and other performance enhancing hardware that would adversely affect timing predictability. When attempting to meet design constraints such as battery lifetime, determining the worst-case energy consumption of software would be of interest, and thus determining the impact of data operands on dynamic energy consumption.

\section{Formalising the circuit switching problem}

As illustrated in the previous section, the matter we consider is the amount of energy caused by circuit switching, specifically the switching occurring on the output datapath in a processor. Here, we formalise our problem, which we name the "Circuit SWitching Problem" (CSWP), discussing its limitations and generality. Our objective is to take a program, determine the maximum amount of output datapath switching activity that can occur in that program, and, in the process, find the program input that triggers it.

Because we are only concerned with the amount of circuit switching that can occur due to operand values, we choose to limit the problem and avoid any facility for varying the length of a program in this formalisation, i.e. the number of instructions executed. A CSWP program thus cannot have any branch instructions or conditional execution ability: it corresponds closely with a trace of a general program execution, or a general program that has been unrolled and all conditional branches eliminated. Dealing with programs of varying length would involve searching different paths through the program, and the variation of energy consumed by static 
power would become a significant consideration. We chose to work with fixed-length CSWP programs to maintain focus on switching in data operands.

Formally, we consider a CSWP program, $X$, to be a finite sequence of $n$ instructions, $x_{i}$, such that $X=x_{1}, x_{2}, \ldots, x_{n}$. Each instruction is a 3-tuple $\langle m, i, o\rangle$, where $m$ is a mnemonic $m \in M$, $i$ is a set of input operands (discussed below), and $o$ is an output operand. Both inputs and outputs (discussed further below) are considered to be bit-vectors of width $w$.

A CSWP program executes on an abstract machine with a monotonically incrementing program counter, an infinite number of registers, and a memory store of finite size. Memory is considered to be an array of size $2^{w}$ with each memory cell a bit-vector of width $w$. For each instruction $x_{i}$ in the CSWP program the machine takes the input operands, computes an output according to the function of the instruction mnemonic, and writes the result to the output operand. The objective function of CSWP is then to compute:

$$
\sum_{i=1}^{n-1} h\left(o_{i}, o_{i+1}\right)
$$

where $h$ is a function computing Hamming distance between two values, i.e. the output values of each subsequent instruction, corresponding to the output datapath of the abstract machine.

Each mnemonic $m \in M$ represents a function over the input operands, resulting in a single output result. In line with the constraints detailed above, CSWP programs only perform arithmetic and logic computations, mapping input operands to an output. There are no branch mnemonics, neither are there any instructions that induce side effects of any form (such as changing some state or the program counter). We do not define a set of mnemonics that a CSWP program may use, however for the purposes of this paper we write listings using standard RISC mnemonics such as add, sub, ldr, mov [14].

Each input operand is permitted to be one of four classes of sources:

- Free inputs, which we denote with the text free.

- Constant values, which we write in hexadecimal, e.g. Ox1.

- A memory access to a fixed address $m[x]$, with $x$ the address.

- The output result of a prior instruction, written $o_{i}$, where for the current instruction $x_{j}$, $i<j$.

The value of every input is always a bit-vector of width $w$. Free inputs may take any value, likewise constants may only have one value, defined in the instruction being executed. Memory accesses evaluate to the contents of a memory cell, but for simplicity we only permit the addressing of fixed memory addresses. Prior output operands correspond to the output of each instruction being written to one of the registers, which may then be read as an input to another instruction.

All instructions are considered to have an output of bit-width $w$, i.e., they all write some value to the output datapath of the machine. A nop (no-operation) instruction would be any instruction that repeats the output value of the previous instruction, causing no switching activity on the output datapath. Outputs may optionally be written to a memory cell $m[x]$, where $x$ is a fixed address for the output value to be written to. In this circumstance, the output value may still be referred to as $o_{i}$, as a store to memory still causes the bits in the machine's result datapath to flip.

This formalisation has a number of limitations, most notably that without an infinite data store or ability to programmatically address it, it is not Turing complete. Given that our aim is to find the maximum switching for a particular path through a general program, this is a 
suitable restriction. The formalisation does not correspond to a particular machine, although with additional restrictions it may correctly model the execution trace of existing processors. The memory array may be considered to be superfluous given the lack of complex addressing, however it provides a useful mechanism for illustrating our examples through the rest of this paper.

We observe that CSWP is in class NP, as one may easily check the validity of a solution. Given the CSWP program and an input valuation for each free input, we can simulate the program with the given inputs, counting the number of bit flips at the same time. The complexity of this process scales linearly with the number of instructions, $n$.

\section{Reducing MAXSAT2 to the circuit switching problem}

To demonstrate that the CSWP is NP-hard, we must reduce any NP-hard problem to CSWP in polynomial time. For this, we turn to the MAXSAT problem, which is known to be NP-hard [3]. Specifically, we work with the MAXSAT2 variant, where each clause is limited to having at most two literals. Despite 2SAT being solvable in polynomial time, MAXSAT2 is still known to be NP-hard [40].

We reduce MAXSAT2 to CSWP by simulating MAXSAT2 in the switching activity of an instruction sequence, where the input that causes the maximum amount of circuit switching corresonds to an assignment to the Boolean variables that causes the maximum number of clauses to be satisfied. The reduction is illustrated in Algorithm 1, which takes the number of Boolean variables and the set of clauses as input, and outputs a CSWP program that simulates MAXSAT2. Here, we assume that the function PrintInsn causes a CSWP instruction to be emitted from the algorithm, with the instruction mnemonic, set of variables, and optional output destination as its respective arguments. The return value identifies the output operand of the instruction.

First, we read a series of free input values, and for the moment we assume they lie in the range $[0,1]$, i.e. represent true or false in the lowest bit of the otherwise zero bit-vector. We consider each of these values to be an assignment to a Boolean variable in the MAXSAT2 problem. Each bit, and its complement, are stored to a location in memory. This creates an array of values corresponding to the truth of each literal. At the end of this process we insert a mov instruction that loads a zero value, for the purpose of resetting the value on the output datapath to zero. The net effect is that for each Boolean variable read, a constant amount of switching activity occurs. Consider each value the free variable may have:

1. True: Reading the input switches the lowest output datapath bit to on, the subsequent xor switches it to off, and the final mov causes no switching.

2. False: Reading the input causes no switching, the xor switches the lowest output datapath bit to on, and the subsequent mov switches it back to off.

Thus, for each Boolean variable read, the CSWP program always causes two bit flips.

We then proceed to use the memory region prepared with literal valuations to simulate the MAXSAT2 problem. We assume a mapping between each literal of the Boolean variables and the address of its valuation in the memory array, and use the function LitToMemAddr to translate from literal to memory address. Then, for each clause, we produce an instruction sequence that loads each literal valuation using the constant-switching technique used to read free inputs. Once the literals are loaded, they are or'd together, after which the output datapath is loaded with zero again.

The CSWP program produced by Algorithm 1 has both a constant and data dependent portion of switching activity. Two bit-flips occur for each Boolean variable in the input MAXSAT2 


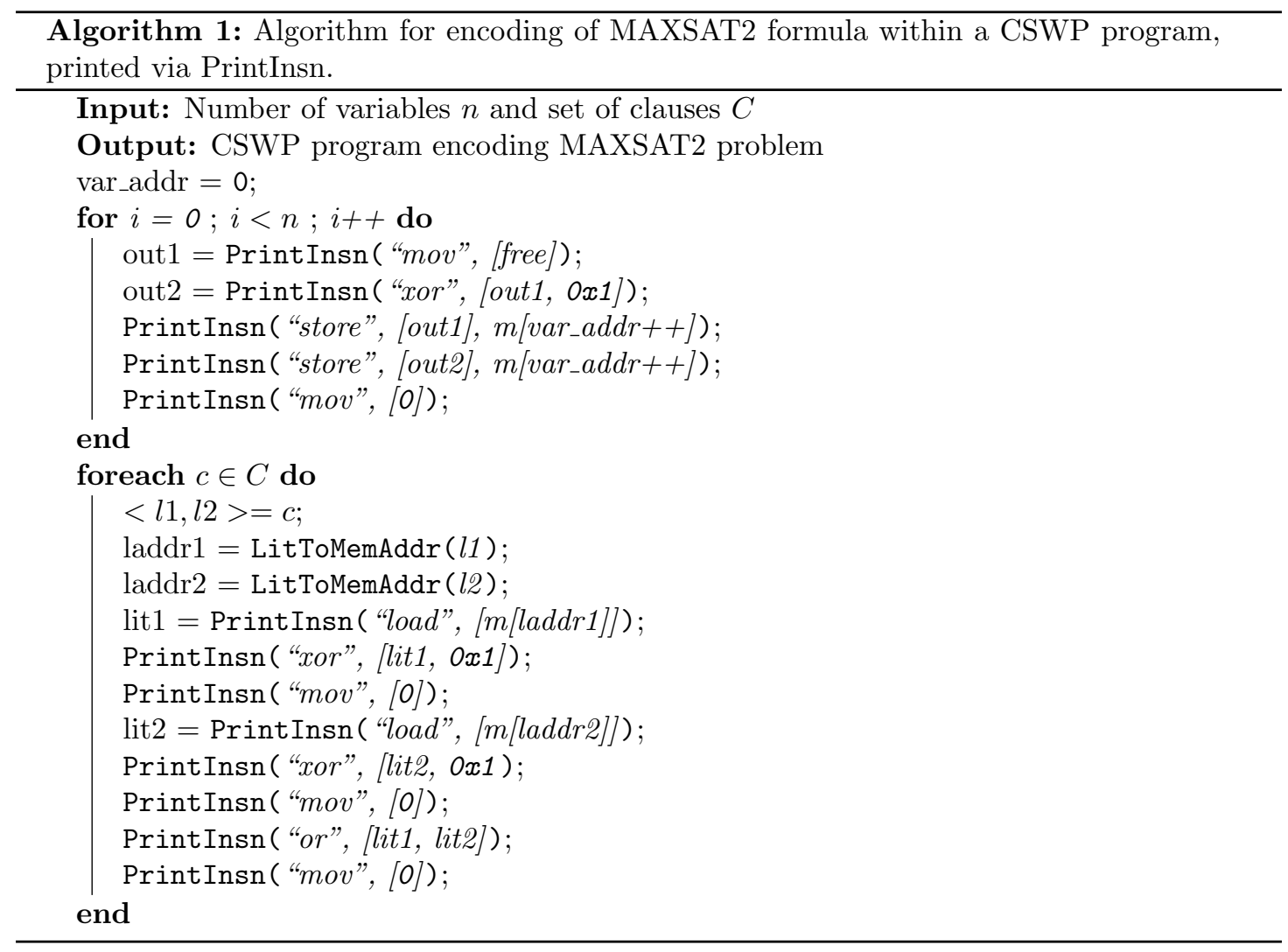


problem, and four for each clause. The switching activity from the or instruction, however, directly corresponds to the satisfiability of the clauses: if a clause is satisfiable (i.e., one of the literals is true) then the or and following mov will cause two additional bit-flips. If a clause is not satisfiable, the same instructions will cause no switching. As a result, the maximum amount of switching in the program is caused by the maximum number of clauses being satisfied. The assignment to the free variables which causes this is also an assignment to the Boolean variables of the MAXSAT2 problem that causes the maximum number of clauses to be satisfied. As a result, CSWP must be at least as hard as MAXSAT2 (i.e. NP-hard). As we know CSWP is also in class NP (Section 4), CWSP is NP-hard.

The assumption that free variables are either one or zero is to simplify the presentation: a similar technique to balance switching can be used to translate any input word into one or zero, with constant switching. For example on a machine with a condition status register, one may compare the input register with zero, exclusive-or the entire input register with an all-ones value, then store the comparison result flag to a general register and continue with the procedure above.

We observe that the reduction is performed in polynomial time, as it scales linearly with the number of Boolean variables $n$ and the number of clauses, of which there can be at most $n^{2}$.

Given this result, we can conclude that there cannot be an efficient algorithm that solves the CSWP, unless $\mathrm{P}=\mathrm{NP}$. Thus, given that general programs can be unrolled and reduced to a CSWP, it is infeasible to determine the exact worst-case switching due to operand values in a program, defeating energy estimation techniques that would rely on such a model. This corresponds to the first form of WCEC estimate given in Section 2.5. However, given such a limitation, there could still be algorithms that approximate the worst-case switching to a certain degree of accuracy, allowing worst-case switching to be narrowed down to a small range of values. We address this in the next section.

\section{Inapproximability}

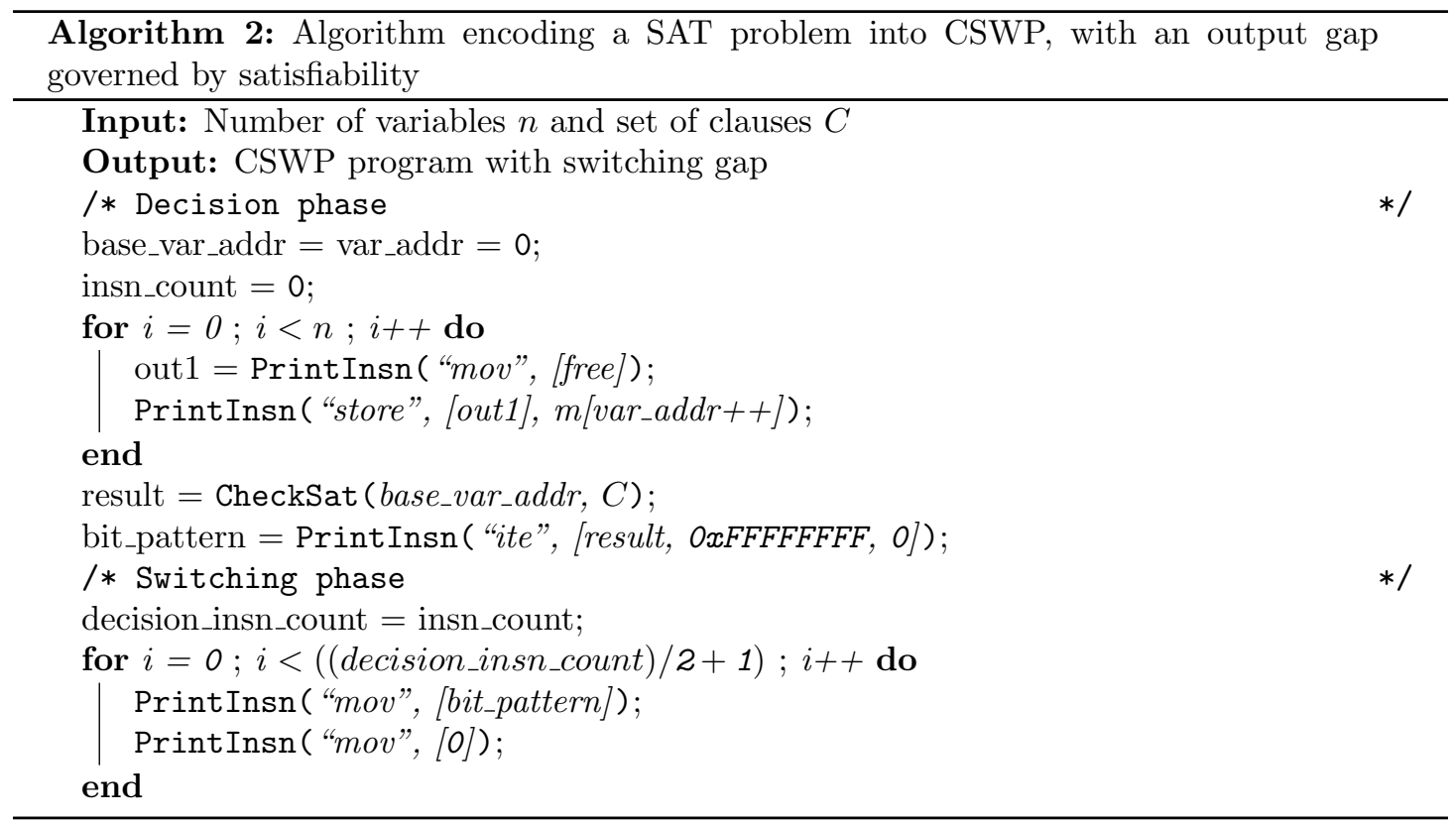


Having shown that CSWP is NP-hard, we will now show that it also cannot be approximated to any useful factor, i.e. the second form of WCEC estimate in Section 2.5 is infeasible. We demonstrate that there is no constant $\varepsilon$ for which an approximation factor of $1-\varepsilon$ can be achieved, and then that polynomial approximation factors also cannot be achieved. Intuitively, this is because each bit flip caused by the program is the result of an arbitrary computation, meaning there is no structure to the combinatorial problem that one can generally rely upon when constructing an approximation.

Formally, we demonstrate CSWPs inapproximability using a gap introducing reduction [40] from SAT to CSWP. Such a reduction transforms an NP-complete decision problem into an NPhard optimisation problem, with a quantity (the "gap") of the feature being optimised governed by the truth of the decision problem. By demonstrating such a gap, one shows that a portion of the NP-hard problem cannot be approximated in polynomial time, as the approximation algorithm would have to solve an NP-complete problem in the process.

In the context of CSWP, we demonstrate that for any instance of SAT problem $p$, we can reduce it to a CSWP program $q$ where a portion of the switching activity is governed by the truth of whether $p$ is satisfiable. The transformation is illustrated in Algorithm 2, which we divide into two discrete portions: the decision phase, and the switching phase. We use the same functions as in Algorithm 1, with the modification that the PrintInsn function increments a counter, insn_count, for every instruction printed.

Throughout the decision phase, we are not concerned with the switching activity that may occur, and do not seek to control it, in contrast with the previous algorithm. We begin by reading $n$ free variables, which for now we assume to be bit-vectors with either zero or one in the least significant bit and all other bits zero. These free variables are stored to fixed addresses in memory. We then pass the address of the variable valuations and the SAT clauses to the CheckSat function, which emits a CSWP program that evaluates the clauses over the Boolean variables stored at base_var_addr, and returns an output operand identifying whether the assignment satisfied the clauses. Significantly, we do not seek to define how Checksat checks the satisfiability of the clauses, we only assume that it achieves it in a number of instructions polynomial in $n$, the number of Boolean variables. We know that SAT is in NP, so due to complexity theory we also know an assignment can be verified in a polynomial number of instructions. ${ }^{1}$ We then produce an output, bit_pattern, using an "if-then-else" instruction that evaluates to zero if the Boolean variables do not satisfy the clauses, and has all bits set if they do.

For the switching phase, the CSWP instruction counter, insn_count, is read to learn how many instructions there are in the decision phase of the CSWP program. We then emit a pattern that repeatedly loads the variable bit_pattern and then zero. The effect of this is to produce a phase in the program that causes a large amount of switching if the SAT problem $p$ was satisfied; and to not if it was unsatisfiable. In this sequence, a satisfying assignment will cause the switching phase to flip every bit in the result datapath, every instruction; while no switching will occur otherwise.

We have thus introduced a gap in the switching activity of the CSWP program $q$, that is governed by whether the SAT problem $p$ is satisfiable or not. We use the length of the decision phase of the program to ensure that the switching phase is at least the length of the decision phase, plus one or two instructions. This ensures that, regardless of the amount of switching in the decision phase, the switching phase dominates the switching activity of the program. When solving CSWP, if the SAT problem $p$ were satisfiable, then the maximum amount of switching would include the switching phase, and the CSWP solver would be obliged to yield an input to the program that satisfied the reduced SAT problem. If $p$ is unsatisfiable, it would instead yield whatever input maximised the switching in the decision phase.

\footnotetext{
${ }^{1}$ We note that, as the inputs to CheckSat are free variables, we are essentially modelling a SAT solver.
} 
We use the size of the gap to demonstrate that CSWP cannot be approximated. In the previous example the switching phase constitutes at least $1 / 2$ of the possible switching activity: if one possessed an algorithm to approximate such a CSWP program to within a factor of $1 / 2$, then it would be obliged to activate the switching phase of any CSWP program reduced from a satisfiable SAT formula, thus acting as an oracle for an NP-complete problem. Under the $\mathrm{P} \neq$ NP assumption, such an algorithm does not exist.

Furthermore, we are able to extend this result to any constant factor. For any value of $\varepsilon$ and SAT instance $p$, take the desired approximation factor $f=1-\varepsilon$ and set the length of the switching phase to be declen $\times(1 / f)$, where declen is the number of instructions in the decision phase of CSWP $q$. Such a program will have a gap of at least $1 / f$ times the decision phase, that depends entirely on the satisfiability of $p$, and thus cannot be approximated. One need not limit this approach to a constant factor either: one may instead compute $f$ to be some factor that is a polynomial function of the size of SAT problem $p$, for example $n^{2}$, and achieve the same result. This shows that there can be no useful approximation factor for CSWP.

The safety of this result depends on the reduction to $q$ being polynomial in the number of variables $n$ in $p$. Introducing the variables of $p$ scales linearly with $n$, checking the satisfiability of a particular assignment is known to be checkable in polynomial time, and the evaluation of the result into bit_pattern is constant-time. The decision phase is thus a polynomial reduction. The switching phase is controlled by the length of the decision phase (which is polynomial), but also the desired approximation factor. Provided the approximation factor is polynomial, the full reduction is also polynomial.

The assumption in Algorithm 2 that free inputs are only zero or one is again to ease presentation: the precise value is irrelevant so long as the switching phase accounts for the maximum amount of switching it can cause. The only requirement is that a sufficient number of free bits are supplied to CheckSat to represent the free inputs to the SAT problem $p$.

\section{Discussion}

We consider here the scope of these results with regards to the hardware for which dynamic operand energy should be a consideration, and the implications of these results for WCEC analysis.

\subsection{Hardware scope}

Determining a program's switching activity caused by operand values is NP-hard, therefore calculating the worst-case dynamic energy for a program in a way that accounts for its input data set is infeasible. Our result is relevant when the cost of dynamic power is dominated by switching in the output datapath. Clearly, the exact cost of such switching will vary between processors, however our result may be used as a basis for demonstrating that calculating the switching in other components of the processor is also infeasible. For example, because all inputs to instructions are inevitably a direct input or the result of some other instruction, it is reasonable to assume that it is NP-hard to estimate the switching activity of input operands too.

\subsubsection{Processor architecture considerations}

The simplest processor architectures, such as deeply embedded AVR or ARM M0 that might be found in low power IoT scenarios, feature few additional components that would make further contributions to dynamic energy consumption. However, more complex architectures, present in larger IoT and embedded systems, introduce extra contributions. 
For example, data caches will contribute dynamic energy too. First, in the computations they perform to decide upon an outcome, for example determining if a memory address is present in cache. Second is the cost of that outcome, for example the subsequent request to a higher-level cache or main memory. These also depend on program inputs to an extent, but are not modelled by our CSWP formalisation. Other processor components may contribute dynamic energy that is not affected by the inputs to a program. The switching associated with instruction logic (decode, functional unit activation, instruction cache) may contribute dynamic energy regardless of the program input.

Features in some processors, such as out-of-order execution may also defeat our analysis. The circuit switching cost is still present, and its determination will still be NP-hard, however it may occur in an unpredictable fashion that depends on a processor-internal unobservable instruction execution schedule.

Energy saving mechanisms such as power- and clock-gating may also reduce the impact of dynamic energy due to operand values. However, if present, the extent of their effect will be dependent upon the architecture design as well as the technology with which it is manufactured. Coarse-grained mechanisms such as sleep states may also warrant attention, although these may need to be accounted for differently if they are directly controlled by software.

\subsubsection{System-level considerations}

Considering again a cache hierarchy, difference in its dynamic power contribution due to the cost of a hit versus the cost of a miss is likely to be higher than the range of dynamic power due to operand values within the processor core. If this cache cost is added to the dynamic energy of the processor core, then the dynamic variation due to operand values is now a much smaller proportion of total energy consumption.

In such a system, it remains infeasible to determine a worst-case due to operand values as per our CSWP formalisation. While the impact of this may be lower, there remains a level of uncertainty that risks compromising the safety of any energy consumption assumptions that are made when modelling is performed. A mitigation strategy, such as more conservative assumptions of worst-case energy per instruction, must therefore be used. The accuracy of cache modelling must also now be considered, where safety of any energy bounds is compromised if the model does not precisely reflect cache hits and misses for a program.

Beyond caches, systems may have peripherals that eclipse the processor in terms of energy consumption, such as wireless transmitters for communication-heavy IoT applications. In such circumstances, the impact of unsafe or overly-conservative worst-case instruction energy modelling is unlikely to be of concern in the context of energy budgeting.

In summary, it is essential to assess the proportion of energy consumed in the system by the processor, and its dynamic consumption due to operand value, to determine if CSWP should be a concern. This is primarily why the focus of this paper has been upon processors for deeply embedded systems.

\subsection{Implications for WCEC analysis}

\subsubsection{No guarantees}

The infeasibility result for estimating dynamic operand energy over time renders the first two forms of WCEC estimation discussed in Section 2.5 infeasible. Further, it prevents the construction of an instruction level energy model that identifies an accurate worst-case switching cost for each instruction in a given program. Existing techniques that apply WCEC analysis $[15,23,11,22,9,41]$ to software can thus never have an energy model that accurately accounts for worst-case achievable dynamic energy of the given computation. 
We are still left with the coarse over-approximation WCEC estimation techniques. Such techniques provide a safe upper bound but no relationship between that bound and the true WCEC, and our result shows that any such relationship would be infeasible to calculate. For example, calculating the absolute maximum operand switching cost for an execution in the manner of [15] and [41] would be sufficient. The over-approximation inherent with this approach will not yield a tight bound. For example, on the XMOS XS1-L, with dynamic energy contributing $42 \%$ of energy consumption, one would have a similarly sized amount of potential over-approximation regarding the energy consumption of any execution. The expected over-approximation would therefore be somewhere within this range, likely towards the middle if an average case is assumed.

\subsubsection{Alternatives}

Viable techniques for estimating dynamic energy consumption can come from a variety of fields: in particular, statistical methods [30] may be effective for determining the distribution of energy consumption under normal operation. Such a model may be used by assuming that the most energy the program can consume occurs only $1 \%$ of the time, and taking the energy value corresponding to that probability as the program's worst-case energy consumption. This does not provide a safe upper bound on the program's energy consumption as it is based on normal operation. However, on the balance of probability it is very likely to present an upper bound. Depending on the use case, such a bound may be more useful in making energy consumption of software transparent to developers than gross over-approximation.

Another alternative is to refine coarse over-approximations: simply assuming maximum switching activity for the whole length of the program yields a likely very inaccurate upper bound. Further techniques such as static analysis or abstract interpretation could reduce this inaccuracy. For example, if one can determine the integer interval of a variable, then one can potentially bound the amount of switching between adjacent instructions, and thus determine the maximum switching of a specific instruction sequence to be lower than its absolute maximum.

In all circumstances, alternative estimation techniques will posses some level of unquantifiable over-approximation, unsafeness or incompleteness, otherwise they will be NP-hard as proven in this work.

\section{Conclusions and future work}

In this paper we have considered the energy consumption in a processor that can directly be attributed to the data or inputs to the software being executed, and demonstrate that the general analysis of circuit switching in processor datapaths - the "circuit switching problem" - is NP-hard. Further, we demonstrate that there is no efficient approximation algorithm for the circuit switching problem to any constant or polynomial factor. We conclude that only the coarsest of estimation techniques can be used in the analysis of worst-case energy in polynomial time. This limitation introduces an uncertain amount of over-approximation in the gap between the true WCEC and the estimated WCEC.

We consider alternate techniques and questions that one could pose that do not amount to worst-case analysis but do provide an estimate of how large the worst case could be, and how they can contribute to understanding software energy consumption.

In the future we believe that work is best focused on statistical methods of modelling program energy consumption, or otherwise characterising the way in which software operates. Similar efforts are being made that model the WCET problem probabilistically [6], for example using extreme value theory (EVT) [24], although recent research suggests EVT may not be completely 
applicable to WCET in general [21]. Critically, we cannot continue to think in terms of a mathematically proven "worst-case" energy consumption, but must instead turn to other methods for energy consumption analysis that may not be sound or accurate, but are at least feasible.

Further exploration of the scope of data-dependent energy would also benefit WCEC. For example, whilst it is intuitive that a cache miss has a higher energy cost than a cache hit by a significant margin, how large a contribution to dynamic power is made by the address and data values on the buses in the memory hierarchy? This would establish whether caches can present energy anomalies of a similar nature to already studied timing anomalies. In addition, this could further address how tight a safe WCEC bound can be in more complex microarchitectures.

\subsection{Acknowledgements}

We would like to thank David May, Benjamin Sach, Kyriakos Georgiou and James Pallister for their insights into and motivation of this work. The research leading to these results has received funding from the European Union 7th Framework Programme (FP7/2007-2013) under grant agreement no 318337, ENTRA - Whole-Systems Energy Transparency; and grant agreement no 611004, ICT-Energy.

\section{References}

[1] ARM. Arm cortex-m programming guide to memory barrier instructions. Technical report, ARM, 2012.

[2] Giuseppe Ascia, Vincenzo Catania, Maurizio Palesi, and Davide Sarta. An instruction-level power analysis model with data dependency. VLSI DESIGN, 12(2):245-273, 2001.

[3] Armin Biere, Marijn Heule, Hans van Maaren, and Toby Walsh. Handbook of Satisfiability: Volume 185 Frontiers in Artificial Intelligence and Applications. IOS Press, Amsterdam, The Netherlands, The Netherlands, 2009.

[4] Paulo Francisco Butzen and Renato Perez Ribas. Leakage current in sub-micrometer cmos gates. Technical report, Universidade Federal do Rio Grande do Sul, 2006.

[5] Aaron Carroll and Gernot Heiser. An analysis of power consumption in a smartphone. In Proceedings of the 2010 USENIX conference on USENIX annual technical conference, USENIXATC'10, page 21, Berkeley, CA, USA, 2010. USENIX Association.

[6] Francisco J. Cazorla, Tullio Vardanega, Eduardo Quiñones, and Jaume Abella. Upperbounding Program Execution Time with Extreme Value Theory. In Claire Maiza, editor, 13th International Workshop on Worst-Case Execution Time Analysis, volume 30 of OpenAccess Series in Informatics (OASIcs), pages 64-76, Dagstuhl, Germany, 2013. Schloss Dagstuhl-Leibniz-Zentrum fuer Informatik.

[7] Lokesh Chandra and Sourav Roy. Estimation of energy consumed by software in processor caches. In 2008 IEEE International Symposium on VLSI Design, Automation and Test (VLSI-DAT), pages 21-24. IEEE, April 2008.

[8] Ana T. Freitas, Horcio C. Neto, and Arlindo L. Oliveira. On the cmoplexity of Power Estimation Problems. 2004.

[9] Kyriakos Georgiou, Steve Kerrison, and Kerstin Eder. On the value and limits of multilevel energy consumption static analysis for deeply embedded single and multi-threaded programs. Technical report, University of Bristol, 2015. 
[10] Ricardo Gonzalez, Benjamin M Gordon, and Mark A Horowitz. Supply and threshold voltage scaling for low power cmos. IEEE Journal of Solid-State Circuits, 32(8):1210-1216, 1997.

[11] Neville Grech, Kyriakos Georgiou, James Pallister, Steve Kerrison, Jeremy Morse, and Kerstin Eder. Static analysis of energy consumption for llvm ir programs. In Proceedings of the 18th International Workshop on Software and Compilers for Embedded Systems, SCOPES '15, pages 12-21, New York, NY, USA, 2015. ACM.

[12] Hadi Hajimiri, Kamran Rahmani, and Prabhat Mishra. Efficient peak power estimation using probabilistic cost-benefit analysis. In VLSI Design (VLSID), 2015 28th International Conference on, pages 369-374, Jan 2015.

[13] Rehan Hameed, Wajahat Qadeer, Megan Wachs, Omid Azizi, Alex Solomatnikov, Benjamin C. Lee, Stephen Richardson, Christos Kozyrakis, and Mark Horowitz. Understanding sources of inefficiency in general-purpose chips. Proceedings of the 37th annual international symposium on Computer architecture - ISCA '10, page 37, 2010.

[14] John L. Hennessy and David A. Patterson. Computer Architecture, Fifth Edition: A Quantitative Approach. Morgan Kaufmann Publishers Inc., San Francisco, CA, USA, 5th edition, 2011.

[15] Ramkumar Jayaseelan, Tulika Mitra, and Xianfeng Li. Estimating the worst-case energy consumption of embedded software. In Proceedings of the 12th IEEE Real-Time and Embedded Technology and Applications Symposium, RTAS '06, pages 81-90, Washington, DC, USA, 2006. IEEE Computer Society.

[16] David S. Johnson. Approximation algorithms for combinatorial problems. In Proceedings of the Fifth Annual ACM Symposium on Theory of Computing, STOC '73, pages 38-49, New York, NY, USA, 1973. ACM.

[17] David Kearney and Neil W. Bergmann. Performance evaluation of asynchronous logic pipelines with data dependent processing delays. In Asynchronous Design Methodologies, 1995. Proceedings., Second Working Conference on, pages 4-13, May 1995.

[18] Steve Kerrison and Kerstin Eder. Energy modeling of software for a hardware multithreaded embedded microprocessor. ACM Trans. Embedded Comput. Syst., 14(3):56, 2015.

[19] NS Kim, T Austin, D Baauw, and T Mudge. Leakage current: Moore's law meets static power. Computer, pages 68-75, 2003.

[20] Yebin Lee and Soontae Kim. DRAM energy reduction by prefetching-based memory traffic clustering. Proceedings of the 21st edition of the great lakes symposium on Great lakes symposium on VLSI - GLSVLSI'11, page 103, 2011.

[21] G. Lima, D. Dias, and E. Barros. Extreme value theory for estimating task execution time bounds: A careful look. In 2016 28th Euromicro Conference on Real-Time Systems (ECRTS), pages 200-211, July 2016.

[22] Umer. Liqat, Kyriakos. Georgiou, Steve. Kerrison, Pedro. Lopez-Garcia, John P. Gallagher, Manuel V. Hermenegildo, and Kerstin. Eder. Inferring Parametric Energy Consumption Functions at Different Software Levels: ISA vs. LLVM IR, pages 81-100. Springer International Publishing, Cham, 2016. 
[23] Umer Liqat, Steve Kerrison, Alejandro Serrano, Kyriakos Georgiou, Pedro Lopez-Garcia, Neville Grech, Manuel V. Hermenegildo, and Kerstin Eder. Energy Consumption Analysis of Programs based on XMOS ISA-level Models. In Logic-Based Program Synthesis and Transformation, 23rd International Symposium, LOPSTR 2013, Revised Selected Papers, volume 8901 of Lecture Notes in Computer Science, pages 72-90. Springer, 2014.

[24] F. Longin. Extreme Events in Finance: A Handbook of Extreme Value Theory and its Applications. Wiley Handbooks in Financial Engineering and Econometrics. Wiley, 2016.

[25] Thomas Lundqvist and Per Stenström. Timing anomalies in dynamically scheduled microprocessors. In Proceedings of the 20th IEEE Real-Time Systems Symposium, RTSS '99, pages 12-, Washington, DC, USA, 1999. IEEE Computer Society.

[26] David May. The XMOS XS1 architecture. available online: http://www.xmos.com/published/xmos-xs1-architecture, 2013.

[27] Michael Hsiao et al. K2: an estimator for peak sustainable power of VLSI circuits. Low Power Electronics and Design, 1997.

[28] Pedro Marques Morgado, Paulo F. Flores, and Luis Miguel Silveira. Generating realistic stimuli for accurate power grid analysis. ACM Trans. Des. Autom. Electron. Syst., 14(3):40:1-40:26, June 2009.

[29] James Pallister, Kerstin Eder, Simon J. Hollis, and Jeremy Bennett. A high-level model of embedded flash energy consumption. In Proceedings of the 2014 International Conference on Compilers, Architecture and Synthesis for Embedded Systems - CASES '14, pages 1-9, New York, New York, USA, 2014. ACM Press.

[30] James Pallister, Steve Kerrison, Jeremy Morse, and Kerstin Eder. Data dependent energy modelling: A worst case perspective. In Proceedings of the 18th International Workshop on Software and Compilers for Embedded Systems, SCOPES '17, New York, NY, USA, 2017. ACM. To appear, preprint at http://arxiv.org/abs/1505.03374.

[31] Amisha Parikh, Soontae Kim, Mahmut T. Kandemir, Narayanan Vijaykrishnan, and Mary Jane Irwin. Instruction scheduling for low power. Journal of VLSI signal processing systems for signal, image and video technology, 37(1):129-149, 2004.

[32] Suzanne Rivoire, Parthasarathy Ranganathan, and Christos Kozyrakis. A comparison of high-level full-system power models. In Proceedings of the 2008 Conference on Power Aware Computing and Systems, HotPower'08, pages 3-3, Berkeley, CA, USA, 2008. USENIX Association.

[33] Uwe Schöning and Jacobo Torán. The Satisfiability Problem: Algorithms and Analyses. Mathematik für Anwendungen. Lehmanns Media, 2013.

[34] Yakun Sophia Shao and David Brooks. Energy characterization and instruction-level energy model of Intel's Xeon Phi processor. In International Symposium on Low Power Electronics and Design (ISLPED), number November, pages 389-394. IEEE, September 2013.

[35] Amit Sinha and Anantha P. Chandrakasan. Energy aware software. In Proceedings of the 13th International Conference on VLSI Design, VLSID '00, pages 50-, Washington, DC, USA, 2000. IEEE Computer Society. 
[36] Stefan Steinke, Markus Knauer, Lars Wehmeyer, and Peter Marwedel. An Accurate and Fine Grain Instruction-level Energy Model Supporting Software Optimizations. In Proceedings of PATMOS, 2001.

[37] Lothar Thiele and Reinhard Wilhelm. Design for timing predictability. Real-Time Syst., 28(2-3):157-177, November 2004.

[38] Vivek Tiwari, Sharad Malik, and Andrew Wolfe. Power analysis of embedded software: a first step towards software power minimization. Very Large Scale Integration (VLSI) Systems, IEEE Transactions on, 2(4):437-445, Dec 1994.

[39] Vivek Tiwari, Sharad Malik, Andrew Wolfe, and Mike Tien-Chien Lee. Instruction level power analysis and optimization of software. J. VLSI Signal Process. Syst., 13(2-3):223238, August 1996.

[40] Vijay V. Vazirani. Approximation Algorithms. Springer-Verlag New York, Inc., New York, NY, USA, 2001.

[41] Peter Wägemann, Tobias Distler, Timo Hönig, Heiko Janker, Rüdiger Kapitza, and Wolfgang Schröder-Preikschat. Worst-case energy consumption analysis for energy-constrained embedded systems. In Real-Time Systems (ECRTS), 2015 27th Euromicro Conference on, pages 105-114, July 2015.

[42] Reinhard Wilhelm, Jakob Engblom, Andreas Ermedahl, Niklas Holsti, Stephan Thesing, David Whalley, Guillem Bernat, Christian Ferdinand, Reinhold Heckmann, Tulika Mitra, Frank Mueller, Isabelle Puaut, Peter Puschner, Jan Staschulat, and Per Stenström. The worst-case execution-time problem - overview of methods and survey of tools. ACM Trans. Embed. Comput. Syst., 7(3):36:1-36:53, May 2008. 